\title{
Integrierte Versorgung - ein neues Instrument der Gesundheitspolitik als Chance für Naturheilverfahren und Komplementärmedizin
}

Relativ unbemerkt geblieben ist der Paragraph 140a des Sozialgesetzbuches V, der die integrierte Versorgung beschreibt. Einige Neuerungen haben Hemmnisse, die zu einer mangelnden Rezeption in früheren Versionen geführt haben, abgeschafft. Durch diese Neuerungen wird die integrierte Versorgung zu einem Instrument der Gesundheitspolitik, das innovative Versorgungskonzepte ausserhalb der bereits bestehenden Verträge zwischen Krankenkassen und kassenärztlicher Vereinigung möglich macht. Die Krankenkassen können nämlich bis maximal $1 \%$ ihres Gesamtbudgets - dies ist nach Berechnungen der kassenärztlichen Vereinigungen ein Betrag von etwa 680.000.000 Euro, bezogen auf das Budget von 2002 - ausserhalb der kassenärztlichen Versorgung für die integrierte Versorgung zur Verfügung stellen.

Innerhalb der integrierten Versorgung sollen Anbieter ein Konzept entwickeln, in dem Patienten nicht von einem Anbieter zum anderen geschickt werden, sondern alle Elemente einer guten Gesundheitsversorgung aus einer Hand beziehen. Konkret bedeutet dies, dass die Primärversorgung sowohl mit der Spezial- und stationären Versorgung und idealerweise auch mit Psychotherapie, Physiotherapie und Reha gekoppelt werden soll und von einem Anbieter separat über die Krankenkassen abgerechnet werden kann. Ziel dieser Unternehmung ist es, dem Patienten ein einheitliches Versorgungs- und Behandlungskonzept anbieten zu können und die Kasse von unnötigen Doppelkosten zu entlasten. Dadurch, dass die Versorgung in einer Hand gebündelt wird, werden Leerläufe, unnötige Wege, Doppeldiagnostik und Fehlbehandlungen hoffentlich vermieden. Anbieter der integrierten Versorgung müssen sicherstellen, dass sie die entsprechende Diagnostik und Behandlung aus dem ihnen zugewiesenen Budget selbst bezahlen. Integrierte Versorgung kann krankheitsübergreifend oder indikationsspezifisch geschehen, muss aber ein möglichst breites Spektrum der Anbieter verbinden.

Dies ist nun ein Konzept, das es auch der Naturheilkunde möglich machen würde, aus der Tertiär- oder Sekundärversorgung in die Primärversorgung zu wechseln. Denn anhand dieses Konzeptes könnten Verbünde von naturheilkundlichen oder komplementärmedizinischen Anbietern Verträge mit Krankenkassen abschliessen und Patienten bereits in der Primärversorgung naturheilkundlich behandeln. Es ist ja ein oftmals gehörtes und praktisch nie belegtes Diktum, dass naturheilkundliche oder komplementärmedizinische Versorgung bereits in der Primärbehandlung viele Kosten, Komplikationen und Nebenwirkungen ersparen würde und somit zu einer effizienteren, schonenderen und kostengün- stigeren Behandlung von Patienten beitrage würde. Hier ist nun das politische Instrument, um eine solche Behauptung auch politisch und volkswirtschaftlich effektiv zu demonstrieren. Geschehen müsste Folgendes: Institutionen mit einer guten Infrastruktur, die komplementärmedizinische Versorgung anbieten - z.B. gut erreichbare Kliniken mit Ambulanzen, Universitätsambulanzen, grosse Gemeinschaftspraxen von Komplementärmedizinern, ein bereits fest etabliertes Praxisnetzwerk - könnten sich zusammenschliessen, andere Anbieter, die gleiche therapeutische Konzepte benützen, mit in ein Konsortium nehmen (z.B. Psychotherapeuten, Physiotherapeuten, Rehabilitationsstationen) und ein Konzept erarbeiten, welches dazu führt, dass Patienten bereits in der Primärversorgung komplementärmedizinisch oder naturheilkundlich behandelt werden und bei Komplikationen oder schwereren Erkrankungen nicht einfach wieder aus dem Versorgungssystem entlassen, sondern an eine andere Stelle innerhalb des Systems weiter überwiesen werden. Das Budget hierfür berechnet sich ausserhalb dessen, was die kassenärztliche Versorgung an Kompensationen zulässt.

Der Anreiz für komplementärmedizinische Forschung bestünde nun darin, eine solche integrierte Versorgung mit einem Modellvorhaben zu koppeln. Dadurch wäre die innovative Versorgungsstruktur automatisch mit einer Begleitforschung zu koppeln, die wiederum extrem wertvolle Daten liefern könnte, um der Komplementärmedizin gesundheitspolitisch zu ihrem angemessenen Platz zu verhelfen, sofern sie ihn denn wirklich verdient.

Wir wollen an dieser Stelle alle interessierten Versorger und auch Forscher dazu aufrufen, innerhalb ihrer eigenen Region solche Verbünde zu gestalten und bei ihren Krankenkassen vorstellig zu werden. Wir haben in unserem Institut bereits positive Rückmeldung auf erste Fühlungnahmen erhalten und gehen davon aus, dass Krankenkassen grosses Interesse an kostensparenden, innovativen und kreativen Konzepten haben, die Luft und Bewegung in die stagnierende Gesundheitspolitik bringen können.

H. Walach, Freiburg i. Br.

Referenz:

SGB V, §140, §140a, §140e

Internet:

http://bundesrecht.juris.de/bundesrecht/sgb_5/_140.html http://bundesrecht.juris.de/bundesrecht/sgb_5/_140a.html http://bundesrecht.juris.de/bundesrecht/sgb_5/_140e.html Mitteilungen der Kassenärztlichen Vereinigung zur integrierten Versorgung gemäss \$140a ff. SGB V unter http://www.aeksh.de/shae/ 200305/h035068a.html. 